\title{
Belphégor
}

\section{Le retournement parodique des discours à leurres constants}

Charles Grivel

\section{(2) OpenEdition}

1 Journals

\section{Electronic version}

URL: https://journals.openedition.org/belphegor/1283

DOI: $10.4000 /$ belphegor. 1283

ISSN: 1499-7185

Publisher

LPCM

\section{Electronic reference}

Charles Grivel, "Le retournement parodique des discours à leurres constants ", Belphégor [Online], 16-1 | 2018, Online since 17 July 2018, connection on 19 October 2021. URL: http://

journals.openedition.org/belphegor/1283 ; DOI: https://doi.org/10.4000/belphegor.1283

This text was automatically generated on 19 October 2021

\section{(c) (i) (9)}

Belphégor est mis à disposition selon les termes de la Licence Creative Commons Attribution - Pas d'Utilisation Commerciale - Pas de Modification 4.0 International. 


\title{
Le retournement parodique des discours à leurres constants
}

\author{
Charles Grivel
}

\section{REFERENCES}

Publié à l'origine dans Clive Thomson et Alain Pagès (dir.), Dire la Parodie, New York et Berne, Peter Lang, « American University Studies », 1989.

1 On présente ordinairement la parodie - le parodique - comme un décroché du discours auquel il s'applique. Comme la falsification bien ordonnée d'un langage ou d'un texte. Comme le faux par excellence, moins honorable et moins parlant que ce à quoi il correspond. Ainsi le parodique est-il vu comme un geste scriptural mineur, plus éloigné de la vérité que l'ouvrage ou la personne à laquelle il s'en prend. Il y aurait, d'un côté, le propre et la source, de l'autre, le parodique, descendances et décadences. Comme si le sérieux était plus sérieux que ce qui - parce qu'il s'en détache - nous fait rire. Apparemment rire.

2 Je propose de renverser le point de vue et la machine. D'explorer le nœud que fait le parodique avec sa vérité. Comment du vrai - si l'on peut dire - s'obtient - ou s'arrache par recrudescence des signes, par la bande et la voie la plus indirecte ; un discours est deux discours: celui qu'il tient, celui dont il se retient. Avec refente. Et par humour sans traces aussi.

3 Certes, il est des parodies grosses, clownesques : elles devraient nous intéresser moins que les autres. Que se passe-t-il quand je m'attache au droit fil de mon discours? Il se rompt ou je dévie. Puis-je parler autrement que par le biais? Les intermédiaires sont les aides de ma bouche (mes autres culturels : ma bibliothèque est pleine de ceux que je conspue, ayant mis leurs mots dans ma langue); les intermédiaires sont mes ponts naturels (ma parole est tout cela que je vole). A écrire, du reste, un auteur verse à la parodie de soi-même : comment ne recommencerait-il pas? 
Comment ne faudrait-il pas qu'il recommence? Comment ne changerait-il pas juste un peu avant de mourir bien? Que les signes de la reprise soient enfouis ou qu'ils émaillent la surface du discours ne fait rien à l'affaire: Duras fait du Duras, Ponson aussi, Jean Ray bien pis encore - mais, à tout prendre, pas plus que chacun. Je dénigre à mesure que j'avance. Or, il me faut cacher ça : vous avouerai-je vraiment que je ne suis pas sérieux?

5 Le fait parodique dénonce ainsi le décollement naturel et nécessaire de la parole d'avec elle-même. Le camouflage, en rire, de ce décollement. L'écriture verse au parodique; les parodieurs replâtrent en faisant croire, un peu plus, que la parodie vient après, qu'elle est un excès extérieur mineur, et aussi mensonger; les parodieurs se tiennent dans l'axe du système.

6 Soit un texte: je propose de le penser immédiatement comme son retournement parodique. Condition : qu'il prenne place dans une série (comment ne pas satisfaire à cette condition ?). Parodiant et parodié figurent en un, à même le texte, sans vérité possible aucune, et pourtant (pensons à Jarry)... L'humour fait partie de la proposition. Indétachable, insaisissable. Elle se tient là.

\section{Introductif}

7 Parodie: des «deux ou trois choses que je sais d'elle». Je convie à quelques observations. Sur pièces. A vrai dire, une chose me frappe : un certain ... tassement de notre savoir à son propos. Le petit - singe à la porte ${ }^{1}$ - veut-il entrer ou veut-on le mettre dehors? - lui aussi, mieux que d'autres - offre un excellent panorama de ce qu'il est possible, de comprendre du fait parodique: ce n'est pas énorme. Notre savoir se restreint à quelques vérités basiques, intéressantes, mais primitives - celles du singe, justement. On se demande si ces vérités sont applicables, à défaut de quoi et en produisant quel reste. Or, c'est le déchet du savoir que l'on possède qui devrait nous intriguer.

8 Applicabilité des théories du fait parodique: la singularité de tout ce qui m'entoure, texte ou pas, me frappe. Les grandes assertions simples dont nous disposons passent à côté de la singularité de ce qui me frappe. C'est dommage. Qu'on me comprenne bien : je ne répudie ce que notre savoir retient, actuellement, du fait parodique ; je dis qu'en toute connaissance de cause, il faudrait oser passer outre - et creuser : creuser dans le particulier, dans l'insolite, dans le hors-jeu théorique, en dehors du réseau, hormis le cadre préposé, sans association préjugée aucune. Excéder nos idées mêmes - celles-là même que nous ne voyons pas.

9 Si cela se trouve, l'objet ou l'action parodique va s'en trouver dissous. Absous? On va peut-être découvrir que le parodique n'est pas grand chose, se répand en fumée, volatilisé par les questions, fortes, que je lui pose. Un objet de communication étant pour commencer un objet de pensée, tant pis pour lui, tant mieux pour nous ...

\section{Correctif}

10 Je reprends les attendus qu'il me faut, que je vois traîner ici ou là hors cause, et proposerai de méditer, préliminairement, sur trois points principaux, avant d'abattre mes cartes et mes « exemples »- ce sont eux qui comptent. 
11 I. D’abord, généralité du fait parodique : la parodie n'est jamais très loin : je la crois participer de l'essence. En fait, doit-on vraiment distinguer entre un jeu - cette occupation dans le drôle - et ce qui n'en serait pas, «travail » ou « sérieux »? Je le crois mal.

12 Parodier suppose un décideur : quelqu'un qui affirme la réalité de la réjouissance par rapport à la réalité, forcément terne, du fait grave. Or, qui est le décideur ? Quels sont ses objectifs, de quel pouvoir - par qui, d'où - est-il investi ? Ces questions découragent, il me semble, de considérer le fait parodique, pour commencer, comme un fait de genre soumis à toute une panoplie de règles "littéraires" ou simplement rhétoriques. La parodie est générale, logée aussi au sein de ce qu'on n'attendait pas touché par ce ... virus, le discours sain, direct, correct, droit.

13 Ouvrons Nodier pour nous en assurer, au début d'un de ses livres, en réponse à un objecteur trop bien-pensant :

- Eh, monsieur, je vois ce que c'est! encore un mauvais pastiche [que votre livre] des innombrables pastiches de Sterne et de Rabelais ...

- Mauvais, cela vous plaît à dire ... et puis, que diable vous faut-il si vous ne voulez pas des pastiches?

Oserois-je vous demander quel livre n'est pas pastiche, quelle idée peut s'enorgueillir aujourd'hui d'éclore première et typique? ...

(...) Oserois-je vous demander, dis-je, quel auteur est procédé de lui-même comme Dieu, si ce n'est l'auteur inconnu qui s'avisa le lendemain de l'invention des lettres

$\cdots$

C'étoit peut-être Enoch : mais son livre ne s'est pas retrouvé

- C'étoit peut-être Abrahams; mais le Jezirah est apocryphe, et le Saint-Esprit le balaya, comme les faux évangiles, de la table du concile de Nicée

- C'étoit peut-être Mercure, autrement Hermès ou Trismégiste ; mais il n'est pas plus question de cette particularité dans Apollodore que dans le père Gautruche.

Qui s'avisa de tracer pour la première fois ... sur le sable

- Ou sur un rocher

- Ou sur une brique

- Ou sur une tabella d'ivoire enduite de cire vierge

- Ou sur toute autre surface naturelle ou plastique, mais pénétrable et tenace

- Ou sur une feuille de papyrus

- Ou sur la membrane du placenta d'un quadrupède

- Ou sur de la bouillie de chanvre ou de lin, de coton ou de soie, de paille ou d'ortie, étendue, aplatie et desséchée

- Avec un roseau aiguisé

Ou un burin pointu

- Ou un crayon de métal friable

- Ou un fragment de pierre colorée

- Ou une plume d'oie

- De tracer (j'en étois là) quelques lignes verticales ou horizontales

- de bas en haut ou de haut en bas

- de droite à gauche ou bien de gauche à droite

- ou même de gauche à droite et de droite à gauche alternativement, comme cela se pratiquoit dans le Boustrophedon

- Et de s'écrier dans une langue qui est morte avant le déluge : Exegi monumentum! Celui-là (écrivain original, je te salue !) n'écrivit cependant, selon toute apparence, que ce qu'on avoit dit avant lui ; et, chose merveilleuse ! le premier livre écrit ne fut lui-même qu'un pastiche de la tradition, qu'un plagiat de la parole! Une idée nouvelle, grand Dieu! il n'en restait pas une dans la circulation du temps de Salomon - et Salomon n'a fait que dire d'après Job. Et vous voulez que moi, plagiaire des plagiaires de Sterne - Qui fut plagiaire de Swift - 
Qui fut plagiaire de Wilkins -

Qui fut plagiaire de Cyrano -

Qui fut plagiaire de Rebour -

Qui fut plagiaire de Guillaume des Autels

Qui fut plagiaire de Rabelais -

Qui fut plagiaire de Morus -

Qui fut plagiaire d'Erasme -

Qui fut plagiaire de Lucien - ou de Lucius de Patras -ou d'Apulée - car on ne sait lequel des trois a été volé par les deux autres, et je ne me suis jamais soucié de le savoir ...

Vous voudriez, je le répète, que j'inventasse la forme et le fond d'un livre ! le ciel me soit en aide! Condillac dit quelque part qu'il serait plus aisé de créer un monde que de créer une idée. Et c'est aussi l'opinion de Polydore, Virgile et Bruscambille. (23 sq.)

14 Je l'écris : ce n'est point neuf! Je le fais : je le refais, ou ça me refait! A peine est-ce tracé que les signes de reconnaissance fusent de toutes parts, catastrophiquement (catastrophiquement pour votre identité d'auteur). Tout lecteur est sardonique : il ne vous croira pas bien, vous ne lui ferez pas, un goût de déjà vu lui viendra facilement à la bouche, il supposera être indigne de lire sans être frappé - feindre être frappé - des évidences narquoises de la reprise, de la duplique et de l'imitation (comment croire en ce monde ?) «Il est clair, dit Bataille, que le monde est purement parodique » (81) : on n'est pas plus ferme de propos! Donc le parodique ne s'isole pas de ce qui lui sert de socle ou de base. Il n'est ni ponctuel, ni générique : on en trouve dès qu'on en cherche, on en a dès qu'on en veut. C'est par hasard si ce n'est pas parodique, semble dire Michel Deguy au seuil de ses réflexions sur l'imitation ${ }^{2}$; tout discours peut être frappé de parodie, comme tout individu d'apoplexie: frappé d'annulation, frappé de vertiges. Obnubilé, surchargé, muté, transcrit. Contrefait et relancé.

Tenons plutôt la parodie comme un acte normal de décomposition culturelle. Tout discours, prise de parole, geste même, émeut de se présenter comme singulier, inédit, neuf, mais se trouve aussi - c'est le paradoxe des sociétés de la consommation - immédiatement recouvert par une interprétation (un acte de reconnaissance) qui le place. Un double mouvement anime le culturel : produire des (soi-disant) singularités, mais réduire ces singularités à de (soi-disant) copies. Parodier, c'est alors, dans l'axe d'une volonté générale de relance fausse, saupoudrer d'un détergent discursif tout original, soit pour s'en saisir, soit pour s'en défaire, simultanément et obligatoirement. L'inédit m'est, culturellement, insupportable quoique nécessaire : je l'envie. Parodier, c'est réduire à la norme commune. Rabaisser. Remettre au pas - dans la bonne voie. J'assimile par la voie détournée d'une reconnaissance autoritaire : le signe imitatif que je décèle m'approprie et me rend ce que l'autre m'avait pris.

Donc 1) ça veut s'exprimer, et ça use pour cela de redites ; 2) ça veut réduire ce qui s'exprime, et ça use aussi, pour cela, de redites. De quelque côté qu'on le prenne, le fait parodique est général.

17 On peut soutenir la même chose - la généralité roborative du parodique - par un autre biais encore. Posons cette définition: parodier, c'est créer de la différence par imitation tempérée (ou inexacte, ou approximative). Or, si cette définition est bonne, tout geste artistique peut être assimilé à la parodie. Je m'abrite ici derrière Chklovski, qui affirme ce qui suit : «L'art est fait pour donner la sensation de la chose reconnue ; le procédé de l'art, c'est le procédé de la représentation [sens fort] «insolite des choses » (cité par Lista, 137). L'insolite, l'écart, la différence, qui est art, fructifie par imitation. Ecrire - 
qui l'aurait cru? - est un travail d'approche : je mine, par adhésion, ce qui me plaît, je reproduis sous ruse ce dont je feins par ruse aussi de me dissocier. Ainsi, trembler ou troubler la reconnaissance engendre un (apparent) produit neuf, par choc en retour, entraîne un plaisir sans exemple - il est mien - qui n'est pas ou plus de simple reconnaissance.

Si cela est vrai, tout texte fait de même, à considérer la série dont il émane : il nous leurre sur un savoir qu'il implique ou appelle, nous l'enlève après l'avoir posé, se retire de son référé, s'écarte du tremplin originel ... Enclos dans l'imitation, mais toujours déjà en passe de rupture.

19 L'écart est général, l'imitation est générale, l'empire des signes est, pour commencer, celui de la parodie. Un ressort caché, qui provient de la position adoptée pour lire, accomplit le texte comme parodie. Grâce à ce ressort, le texte se démet de sa vérité d'expression : rapporté à ce qu'il n'est pas (un autre parodié), il gagne une identité dans l'écart (relativement) subtil qu'il creuse grâce à l'imitation et par-delà celle-ci. Transfert par imitation. Echappée - ou échappatoire - par imitation.

II. Parodier n'est pas refuser, rejeter, abolir. Ne possède pas de caractère annulatoire. On ne l'est que dans les cas simples, inintéressants ici (la moquerie plate a-t-elle de quoi nous arrêter vraiment ?) : parodier n'est pas décroire. Je range la parodie du côté de l'humour, plutôt que du côté de l'ironie. Car l'ironie réserve intacte la place de celui qui en use. Encore une fois, le simple déni par voie d'imitation ne requiert pas mon attention. Pour paraitre drôle, dit le spécialiste, «il suffit que la parodie détruise l'unicité de quelque chose et nous fasse souvenir de la valeur attribuée généralement à cette unicité » (Olbrecht-Tyteca, 99) : si parodier n'était que ça - satire -, je n'aurais rien, décidément, à en dire, épuisée qu'elle serait dans le geste même qui la pose.

21 « La parodie suppose toujours quelque part une naïveté [celle de l'autre !], adossée à un inconscient [celui de son écriture !], et le vertige d'une non-maîtrise, une perte de connaissance [toute réflexion est parodique, toute philosophie aussi !]», dit Derrida (80). Or, remettre à l'autre, cet intime adversaire, l'inconscient du texte, le non-savoir le non-savoir de la non-finalité textuelle -, ne constitue pas, à mon sens, le but de l'opération parodique. Le monde est bourré de petits moqueurs talentueux, capables d'assez bonnes imitations. De gens qui ont, censément, l'identification ou facile ou rieuse : je ne suis pas sûr qu'ils sachent tous bien ce qu'ils font: la prime du plaisir qu'ils me donnent, je la verse à leur actif plutôt qu'à cela qu'ils pensent prendre pour cible.

On peut considérer l'action parodique comme un "rapt d'identité » ou une « dépossession légitime du sens » (G. Idt cité par Vernet, 35); on peut considérer que le parodiant épuise son parodié (tout ce qui s'imite se déprécie) ${ }^{2}$; on peut parodiquement - se simplifier la tâche et dire, façon François Caradec: «La parodie, mes chers enfants, est un genre très ancien qui, sur le mode burlesque et le ton de la farce, tourne en ridicule les idées et la forme d'une œuvre à la mode $»^{3}$. Tout cela autre versant ! - ne doit pourtant pas faire oublier que la parodie est construite en trompe-l'œil. L'objet parodié est un feint objet, la fin de l'opération en cause devant être mesurée à celui qui l'accomplit, à savoir : le sujet parodiant.

Débattre de la parodie entraîne à disputer des leurres: fréquents en littérature, constants pour l'imaginaire. Ça touche où ça ne parait pas correspondre, ça fait précisément là di ça ne parait pas aboutir, etc. 

- revient au sujet frappeur comme une prime qu'il désire s'octroyer. Ainsi, le rire qu'il engendre est-il, pour lui, bénéfice d'identité. Ainsi, Ia jouissance qu'il tire de sa contrefaçon convient-elle et conforte-t-elle sa (débile) personne : pas de parodie sans manque à l'identité du sujet qui l'agit. Guérissement des parodiants ! Cures par le geste et le mot! «Pas de parodie sans 'intention,' sans sujet conscient, sans son intention,» affirme A.-M. Houdebine (63) : « Posture hystérique du sujet parodiant, jouant pour le tiers garant de l'Autre, une/des identité(s) théâtralisant une vérité: un espace de pluralité du sujet (des identifications/désidentifications). " Transfert. Captation de soi par le biais de la mise en scène, leurrée, feinte et fictive, d'un autre posé comme imitable - appréhensible - assimilable et sien. "Copier c'est s'indifférencier, s'immobiliser dans le temps, mais, parodier, c'est, dans la reproduction, produire assez d'écart différentiel pour reconduire la relance de la vie ... « (Montaut, 149). Le discours ou la manifestation de l'autre est mon socle. Je m'érige à sa place, au lieu même où il me parait. « Anverwandlung » : assimilation des substances, vampirisme parodieur.

Arrivé à ce point, j'avancerai neuf thèses :

1. Je parodie ce que je désire sans pouvoir bien me l'avouer.

2. Quel est l'objet du désir parodique ? L'être de l'autre, dont je suis dépossédé. Car l'autre est désirable de ce qu'il m'est dérobé ou refusé. Je Le parodie pour m'en approprier. Le cannibaliser. L'ingérer. Certes. Mais sans me pouvoir faire l'aveu du manque sur lequel, pour rire, je me fonde.

3. Une grande moquerie ambiguë atteint l'autre, comme objet du propre, en raison du désir qu'il me suggère. Le parodier, c'est le placer comme autre et comme mien. Déboitage, décalage. Le tirant à moi par fausse imitation je l'expulse de son lieu, je le désapproprie, je me constitue de ce déni. Le parodier, c'est me libérer du désir qu'il me fait éprouver de son identité modélisante (sublime quoique et parce que déniée). Prométhée parodieur, voleur du feu de personne!

4. Parodier : nier par imitation. Or, l'imitation est un geste d'appropriation. Donc, la négation, qui résulte de l'imitation, n'est rien d'autre que le cache de l'emprunt d'être que je fais. Cassant, vidant l'autre par imitation - imité, il n'est plus lui-même -, je me dote d'un moi inimitable (l'imitateur est en fuite).

5. Il n'y a que des parodieurs jaloux. Parodier, c'est se déprendre du désir de l'autre, c'est s'emparer aussi de cela que l'autre est seul à posséder. La loi de la parodie est la loi du désir : je mets la main sur de l'identité.

6. La parodie est le jeu du moins fort. Je parodie, par ruse, afin de contrecarrer le pouvoir en train de s'exercer. Parodie, revanche des benjamins.

7. La petite parodie est ironique: c'est contrer l'autre «pour soi » dans une insupportable situation d'impuissance (ce n'est plus en chantant, comme à l'âge d'or, que je me déplace). Parodier, c'est troquer un insoutenable moins contre un enviable plus : par dévalorisation de tout ce qu'on touche. Phénomène inflatoire. Mais la parodie bascule à l'humour lorsqu'elle fait intervenir à son point d'aboutissement un sujet non capitalisateur - dépensier - à banque molle ...

8. Le parodieur dévalise, le parodieur déprécie. Avec transfert symbolique : s'assimiler l'être de l'autre par fausse imitation, c'est lui soustraire l'essence et la substance, la vérité, la nature et le propre. Cannibale d'identité, grand dérobeur.

9. Dans un monde (moderne) encombré de produits supposés neufs, « originaux, » vendus pour tels, on ne peut intervenir - faire sa place - donner sa parole à entendre - qu'en s'en prenant au caractère «inédit » de ceux-ci. Alors parodier vise à radier l'originalité inquisitrice - 
quoique fictive - de l'autre. Se substituer - ironiquement - à lui, ou se mettre, avec humour, en quête de nul lieu.

La situation de départ de ce double jeu est alors la suivante : qu'est-ce qui se passe, dit quelqu'un, je ne vous entends pas bien, je trouve que vous parlez à ma place et vois que je manque à ce que vous me communiquez! Comment répondrai-je à cela et comment vous le ferai-je sentir? Parodier, c'est jeter du trouble, comme la seiche son encre, afin de se déplacer sans dommage, insinuer, dissimuler, trouver asile et fondre. Pour mieux frapper, mais surtout pour mieux fuir. Casser les prises, à l'aise et au large, dans les eaux neutres d'un désir sans voix ...Le parodieur, comme le chasseur, - outre image, épuisons les images! - prend soin de ne pas laisser de traces, de peur de devenir luimême, curieux retournement du rêve ! chasse faite et gibier pris, la proie du plus fort que lui (de ses suivants, de ses enfants). Un sujet sachant bien chasser - on dit chasser sur son ancre d'un bateau qui se déplace toutes précautions prises - est un bon gibier sachant bien giboyer : il détale, il fuit, ses directions sont les plus incertaines.

\section{Exploratoire}

J'ouvre des cas : voyons ce qu'ils apportent quant à la compréhension, un peu inversée, du fait parodique. J'en ai de trois sortes : des romans populaires, des nouveaux romans, des pratiques textuelles contemporaines. Ponson du Terrail, Jean Ray, d'un côté; Robbe-Grillet, Renaud Camus, Jean-Claude Pirotte et Denis Roche, de l'autre. Cela fait toute une flotille, mais parodier - goûter le parodique - exige qu'on soit deux, et même plus de deux, dans le temps. Je distingue et j'expose trois machineries parodiques : leur indécidable, leur impondérable - causes aussi bien qu'effets - m'intéresse. En d'autres termes, l'humour qu'elles secrètent comme elles agissent dans la tête lectrice. Avec cela, bien sûr, je ne m'en tiens pas aux valeurs institutionnellement acquises : je brasse les cartes, je mêle le meilleur au pire, le goût de la littérature ne fait jamais rien d'autre, battre mots contre mots jusqu'à ce qu'il en surgisse PLAIRE ...

\section{Le parodique d'excès}

Ou de premier degré. Un texte arrive, qui empire, afin de se faire lire, ceux ou celui qui l'avaient précédé dans la série. Il grossit, par conséquent, chacun des termes, sémantiques ou stylistiques, de la perception.

\section{Thèses}

1. Tout texte d'un genre est toujours déjà potentiellement parodique de lui-même.

2. Tout texte n'est parodique que d'excès. C'est dans la mesure où il donne dans l'hyperbole qu'il se dénote, découvre ou reçoit comme tel.

3. La parodie est une pratique textuelle, autrement dit une lecture.

Condition: inscription des hyperboles dans la substance du texte, le texte doit faire relance, et relance forte, des motifs et de l'aspect de ses prédécesseurs.

\section{Lectures}

Vanda se vit perdue.

Elle n'avait pas d'arme sous la main et avait pour adversaire un de ces hommes aux 
jarrets d'acier qui bondissent comme des tigres.

Mais la femme qui avait si longtemps vécu de la vie de Rocambole ne perdait jamais complètement la tête.

Un miracle seul pouvait la sauver.

Ce miracle, elle le fit sans l'intervention du ciel.

Déjà Sir Nively levait le bras pour frapper, et elle s'était réfugiée à l'autre bout de la chambre ; déjà la lame du poignard étincelait au feu des bougies, lorsque Vanda, par un geste rapide, dégrafa le manteau qui recouvrait son peignoir.

Le manteau tomba.

Le bras levé de l'homme ne retomba point, la bête fauve ivre de carnage s'arrêta piquée au coeur par l'aiguillon de l'amour.

Sir James recula d'un pas.

Et en reculant, il embrassa d'un regard cette beauté hardie.

oh! dit-il en riant d'un rire de tigre, avant que tu ne meures, il faut que ma vengeance soit complète; il faut ... mais il n'acheva pas! Vanda respira; elle avait pour dix secondes détourné la foudre.

Et, à son tour, elle bondit à l'autre extrémité de la chambre et dit en ricanant :

Que m'importe la mort ! que m'importe la honte, pourvu que mon enfant soit sauvé. Ton enfant? s'écria Sir James interdit.

Hé ! oui, mon enfant ! dit-elle.

Puis, avec un rire de hyène, belle de désespoir et d'une ironie farouche :

Croyez-vous pas, dit-elle, que si je n'avais un enfant que Rocambole tient en ses mains, j'aurais obéi à ce forçat?

En même temps, elle se mit à genoux, joignit les mains, passa du rire aux larmes, de la raillerie à l'accent suppliant de la mère et dit :

Faites de moi ce que vous voudrez, tuez-moi ensuite, j'ai mérité mon sort et peu m'importe! Mais sauvez mon enfant, promettez-moi de l'arracher à Rocambole.

Une réaction bizarre s'opérait chez Sir James Nively et son bras avait fini par retomber, toujours armé du poignard, le long de son corps.

Si vous refusez de m'écouter, dit encore Vanda qui se redressa tout à coup, je vous échapperai par la mort. En même temps, elle porta rapidement à ses lèvres une bague qu'elle avait au doigt.

Sir James se laissa prendre à ce geste, il crut que le chaton de la bague renfermait quelque poison foudroyant.

Et ce n'était plus seulement la mort de Vanda qu'il voulait.

(Ponson du Terrail, Les Millions de la Bohémienne, 295-96.)

Les yeux de M. Petito semblaient sortir des orbites. Etait-ce l'effroi ? L'effroi devait y être pour quelque chose, mais aussi l'étouffement qui résultait du mouchoir que Théophraste lui avait enfoncé dans la bouche. M. Petito lui-même était couché sur la table en bois blanc.

Théophraste avait eu le temps et la force invincible de lui lier les poings et les chevilles avec des ficelles. La tête de M. Petito pendait un peu au-delà de la table. A côté de la table et sous la tête de M. Petito, il y avait une jatte que M. Longuet avait placée là pour ne rien salir. Celui-ci les narines palpitantes (c'est ce que Marceline remarqua surtout dans la figure formidable de son mari), avait pris M. Petito par les cheveux, de la main gauche. Dans la main droite, il serrait le manche d'un couteau de cuisine ébréché, qui ne servait guère qu'à ouvrir les huîtres et les bottes de sardines. Les dents de Théophraste grinçaient : Il dit :

- Amène les pavillons !...

Et il entama l'oreille droite. Le cartilage résistait. On entendait, à travers le mouchoir, le hurlement lointain, et tout à fait sourd de M. Petito. Comme M. Longuet était resté en chemise, il semblait, par-derrière, quand on ne voyait pas son visage terrible, un interne penché sur une opération difficile. Marceline, sans force, tomba à genoux. M. Petito tenta un mouvement suprême et le sang de son oreille jaillit à travers la cuisine. Théophraste lâcha les cheveux et lui administra une gifle. - Fais donc attention, disait-il, tu éclabousses partout! 
Comme le cartilage résistait encore, il prit de la main gauche l'oreille droite et, d'un grand coup de couteau ébréché, acheva de l'arracher. Il mit cette oreille dans une soucoupe qu'il avait préalablement déposée sur l'évier.

Et il ouvrit le robinet d'eau dont le jet (dirigé mathématiquement par le brise-jet) alla laver l'oreille de tout le sang dont elle était maculée. Puis il revint à la seconde oreille. Comme Marceline gémissait trop fort, il la fit taire d'un coup d'œil. La seconde oreille fut coupée beaucoup plus vite, sans comparaison, et vraiment quant à moi, j'en suis bien aise, car le découpage de la première oreille avait été une chose affreuse. Il était temps.

M. Petito avait avalé la moitié du mouchoir. Il étouffait. Théophraste retira de la bouche de M. Petito son mouchoir et le jeta dans le panier au linge sale, qui était là, par hasard. Il délia ensuite les chevilles et les poignets du lamentable expert en écriture, et il lui conseilla dans le tuyau de l'oreille puisque l'oreille elle-même avait disparu, de quitter le plus tôt possible son appartement, s'il ne voulait pas qu'il le fit arrêter comme cambrioleur. Il eut encore la précaution de lui envelopper la tête dans un torchon "pour que son sang ne tachât point l'escalier du concierge "; enfin, comme M. Petito, agonisant, se disposait à regagner ses pénates, Théophraste lui mit ses oreilles lavées dans la poche de son veston.

- Vous oubliez tout en route, lui dit-il. Que penserait Mme Petite si vous rentriez sans vos oreilles!

Il referma la porte et, regardant Marceline qui, toujours à genoux, se mourait d'horreur, il essuya le couteau sanglant sur sa manche.

(Gaston Leroux, La Double vie de Théophraste Longuet, 23-25).

On l'avait soulevé pour le poser sur un large tronc d'arbre, et malgré sa main blessée, dont chaque mouvement lui arrachait un grognement de souffrance, le géant Gusher l'y ligotait avec une habileté consommée.

Là, le colis est prêt, dit-il.

Alors, on découpe la mortadelle ! ricana Lehmann.

Et, tout à coup, le détective comprit quel genre de trépas lui destinaient les deux monstres : cinq scies à ruban se mouvaient devant lui en un va-et-vient rapide.

Fritz Lehmann fit un salut comique à sa victime et, avec une politesse affectée, fournit quelques explications.

Ces excellents rubans dentelés vous découpent un tronc dans le sens de la longueur en cinq belles planches. Herr Harry Dickson comprendra donc qu'il aura le même sort que cet arbre inerte : son corps sera découpé en cinq tranches longitudinales. Cela demandera quelque temps, il ne mourra pas tout de suite, mais qu'il ne se gêne pas, au cours de l'opération, pour exprimer ses sensations de vive voix. Il n'y aura que Wolff et moi pour les entendre, et peut-être un ou deux lièvres au gîte, à la lisière de la forêt, mais ce n'est pas eux qui iront le dire ailleurs.

Assez, gronda Gusher, avec ce type-ci, on ne sait jamais à quoi s'en tenir. Plus vite l'ouvrage sera fait, plus vite nous aurons du repos. Si Metzger veut l'avoir entier, il n'aura qu'à recoller les morceaux. Allons !

Harry Dickson sentit l'arbre glisser en avant sous la poussée de ses bourreaux. Il fit un effort pour se libérer, mais ne put bouger d'un pouce.

Le tronc glissait, lentement d'abord, de plus en plus vite ensuite. Une poussière humide faite d'huile et de sciure fraiche vola dans ses yeux ... il entendit le bruit lancinant des scies se rapprocher.

Soudain, l'arbre vibra sous lui : les horribles lames venaient de l'entamer. Harry Dickson ferma les yeux, quelque chose racla les semelles de ses chaussures.

Donnerwetter!

Un double juron retentissant venait d'être poussé à ses côtés.

C'étaient Lehmann et Gusher qui regardaient avec des yeux écarquillés les cinq scies qui venaient de s'arrêter toutes à la fois, au moment di elles allaient commencer leur meurtrière besogne.

Par l'enfer et ses diables, il n'y a plus de courant, mugit Gusher. 
Cela tient à la turbine, gronda Lehmann.

(Jean Ray, Le Mystère de la forêt, 138-139.) public populaire s'en est nourri et cinquante - ou cent ans - plus tard, leurs charmes se déplacent. Je tiens, pourtant, pour acquis: a) que l'hyperbole habite chacun des spécimens - point n'est besoin de grosse analyse pour s'en convaincre ! - et ce sont des textes ou des passages de choc, faits pour férir de grands coups émotifs à grand renfort de représentations exacerbées, évidentes, insupportables et inconvenables; b) qu'une certaine croissance de l'hyperbolisation textuelle est, chronologiquement, de Ponson à Jean Ray - le plus récent - un fait : l'intérêt exige, dans le cadre d'un genre et dans la succession de parution des exemplaires, qu'on en remette (l'exagération seule est propre à combattre la fadeur issue de la répétition) ; c) que la parution - ou même la coparution - pour un lecteur, de la série hyperbolique, constitue le premier des exemplaires qu'il a goûté en objet parodique : c'est à celui-là qu'elle se mesure (cette série), c'est lui qu'elle pille et grossit, c'est lui aussi qui parait - à partir de la position de lecture occupée - comme l'ombre dévalorisée de lui-même. Série fait parodie. Puisque nous lisons, dans la comparaison, exemplaires sur exemplaires au sein d'une bibliothèque hantée par l'innovation. Cela parait toujours déjà parodié-parodiant, objet (masqué) d'une lecture autant décessive qu'appropriative. Ainsi, le premier des textes que je saisis, à cette impossible situation originaire que j'occupe, puisque toujours un autre a précédé, se trouve-t-il immédiatement "parodié, » déplacé par rapport à luimême, inverti : j'entends dans son souffle, déjà, la rumeur des suivants. La preuve de tout ceci est dans le fait que nous rions à la lecture du florilège - au reste, je n'ai fait que rafraîchir les mémoires - qui vient d'être présenté (ce rire n'empêche peut-être pas d'être saisi). Il suffit d'un peu de surenchère, et le texte bascule. Empire des hyperboles: ce que vous forcez, écrivant ou lisant, vous revient risiblement dans la figure; il suffit pour cela d'un autre, d'un miroir, d'un second qui accompagne la parution de ce que vous faites ou dites. Il s'ensuit que le modèle - le premier du genre, le précédent qui fait date - ne peut être surmonté que de façon tempérée (sinon, il verse au parodique). Or, avec la températion de l'écriture, c'est aussi la prime de plaisir qui diminue. Dilemme!

\section{Le parodique de généralisation}

Ou de second degré. Un texte arrive, qui systématise, afin de se faire lire, ceux ou celui qui l'avaient précédé dans la série. Il éclaircit et simplifie, par conséquent, chacun des termes, sémantiques ou stylistiques, de la perception.

\section{Thèses}

1. On considérera l'écriture comme une pratique généralisante: un procédé fait suite au même, de genre identique, qui, lui, était moins «fort »- moins précis, moins appliqué, moins reconnaissable. Moins méthodique. Coupe réglée des écritures. Le savoir - savoir de fabrique - gagne. La généralisation est la loi des évolutions (pestes et pollutions). Je puis en remettre par excès, je le puis aussi par extension, en étalant mon sens, en complétant, parfaisant, parachèvant ma substance : la parodie est un phénomène de rationalisation. Ainsi, a-t-on affaire à un type 1 d'écriture - disons, le nouveau roman standard des années 50, façon La Jalousie - auquel fait suite un nouveau roman de type 
2, de seconde main ou seconde mouture, renchérissant sur l'orthodoxie imposée par les premiers produits du genre et systématisant les procédés en vigueur à ce niveau-là. Il en va de la sorte, quinze ou vingt ans plus tard, pour les romans de Claude Simon postérieurs à Tryptique ou pour les ouvrages que signe Jean Ricardou.

2. Le passage du type 1 au type 2 s'appelle parodie, et cela uniquement dans le cadre de la perception d'un sujet lecteur.

3. Le type 2 constitue la parodie triste du type $1:$ il ne s'avoue pas, ne s'explicite pas, pour son lecteur, avec enjouement, parodique. Pour échapper, sans doute, à la disqualification qui frappe tout phénomène parodique et par horreur du rire, ce vide impénitent des âmes.

4. Il n'y a, proprement, de parodie possible que de romans du type 1. La copie hilarante du second est impropre à produire autre chose qu'un roman - «triste »- du second genre.

\section{Lectures}

S'ouvrant sur une musique romantique, violente, passionnée comme on en entend à la fin des films où 1'émotion éclate (avec tout un orchestre de cordes, bois, cuivres, etc.), le générique est d'abord de type assez classique: des noms en lettres peu ornées, noires sur fond gris, ou blanches sur fond gris; les noms ou groupes de noms sont encadrés de filets simples. Les panneaux se succèdent à un rythme normal, plutôt lent, régulier.

Puis, progressivement les encadrements se transforment, s'épaississent, s'ornent de fioritures diverses qui finissent par constituer comme des cadres de tableaux d'abord plats, puis peints en trompe-l'œil de manière à faire croire à des objets en relief.

Enfin, dans les derniers panneaux du générique, on se trouve bel et bien devant de vrais cadres, compliqués et chargés d'ornements. En même temps, la marge d'image autour d'eux s'est légèrement agrandie, laissant voir un peu du mur ou les tableaux sont accrochés, mur lui-même orné de moulures et boiseries.

Les deux derniers tableaux-génériques, au lieu de constituer des plans séparés, sont découverts progressivement par le coté, dans un déplacement latéral de la caméra, qui, sans s'arrêter sur le panneau lorsqu'il est cadré, continue son mouvement, d'une façon lente et régulière, passe sur une partie du mur comportant seulement des boiseries, lambris, moulures, etc., puis atteint le dernier tableau, portant le ou les derniers noms du générique qui pourrait commencer par des noms secondaires et finir par des noms importants, ou même les mélanger, surtout vers la fin. Ce dernier tableau, comme s'il était vu de plus loin, laisse une marge de paroi très importante autour de lui. La caméra passe de même sur celui-ci sans s'arrêter et continue ensuite son mouvement le long de la paroi.

Parallèlement à l'évolution de l'image, au cours du générique, la musique s'est transformée peu a peu en une voix d'homme, lente, chaude, assez forte, mais avec en même temps une certaine neutralité: belle voix théâtrale, rythmée, sans émotion particulière.

Cette voix parle de façon continue, mais, bien que la musique ait cessé tout à fait, on ne comprend pas encore les paroles (ou on les comprend en tout cas très mal) à cause d'une forte réverbération ou quelque effet du même genre (deux bandes sonores identiques décalées, se rejoignant progressivement jusqu'à devenir une voix normale).

Voix de X : Une fois de plus - . je m'avance, une fois de plus, le long de ces couloirs, à travers ces salons, ces galeries, dans cette construction - d'un autre siècle, cet hôtel immense, luxueux, baroque, - lugubre, où des couloirs interminables succèdent aux couloirs, - silencieux, déserts, surchargés d'un décor sombre et froid de boiseries, de stuc, de panneaux moulurés, marbres, glaces noires, tableaux aux 
teintes noires, colonnes, lourdes tentures, encadrements sculptés des portes, enfilades de portes, de galeries, de couloirs transversaux, qui débouchent à leur tour sur des salons déserts, des salons surchargés d'une ornementation d'un autre siècle, des salles silencieuses ...

(Alain Robbe-Grillet, L'Année dernière à Marienbad. 25-25.)

S'ouvrant sur une musique sérielle, violente, inquiète et irritante, le générique est d'abord de type classique (lettres noires sur fond gris, ou blanches sur fond gris) à cela près que ces lettres ont quelque chose d'inachevé, qui fait que l'on devine les noms plus qu'on ne les lit.

Les pannesoix, qui se succèdent à un rythme normal et régulier, sont encadrés de feuillages. Progressivement, ces feuillages envahissent l'écran, rendant tout à fait impossible la lecture du générique. Les derniers panneaux sont découverts par le côté, dans un déplacement latéral de la caméra, dont le mouvement se poursuit, lent, rectiligne, uniforme, le long d'une sorte de charmille, assez sombre, éclairée seulement par quelques trouées du feuillage. Il n'y a pas de soleil, peut-être même est-ce la nuit.

Parallèlement à l'évolution de l'image, au cours du générique, la musique s'est transformée peu à peu en une voix d'homme, lente, neutre et blanche; même quand la musique a cessé, on ne comprend pas les paroles que prononce cette voix (ou du moins on les comprend très mal).

Voix de X. Une fois de plus, je m'avancerai, une fois de plus, le long de ces charmilles, à travers ces bosquets, ces boulingrins, dans ce jardin d'un autre âge, ce jardin immense, étrange, à la française - sinistre, où des allées interminables succéderont aux allées, - silencieuses, désertes, surchargées de plates-bandes, de buissons, de corbeilles, de berceaux, tonnelles, kiosques, grottes, labyrinthe de contre-allées qui déboucheront à leur tour sur des ronds-points déserts, des orangeries abandonnées, des serres vides ...

Les images qui accompagnent ce texte ne présentent pas d'analogie absolue avec le décor auquel il fait allusion. La caméra ne s'arrête sur rien en particulier, mais la photographie doit avoir durant tout le film un caractère constant : la précision dans le flou.

Voix de X.... où les pas de celui qui s'avancera seront couverts par le crissement du gravier, si consistant, si insistant, qu'aucun bruit de pas ne parviendra à sa propre oreille, comme si l'oreille elle-même de celui qui s'avancera, une fois de plus, le long de ces charmilles, à travers ces bosquets, ces boulingrins, dans ce jardin - d'un autre âge, ce jardin immense, étrange, à la française - sinistre, où des allées interminables succèdent aux allées silencieuses, désertes, surchargées de platesbandes, de buissons, de corbeilles, de berceaux, tonnelles, kiosques, grottes, labyrinthes de contre-allées qui déboucheront à leur tour sur des ronds-points déserts, des orangeries abandonnées, des serres vides, - comme si l'oreille ellemême était pleine de gravier que le mouvement de la marche ferait crisser ...

Le mouvement de la caméra s'achève sur une femme, vingt-cinq à trente ans, belle, mais comme vide (désignons la par la lettre A) assez grande, genre statue.

Voix de X. - Vous serez toujours la même ...

J'aurai l'impression de vous avoir quittée la veille ...

Vous serez toujours aussi belle ... Mais vous ne semblerez guère vous souvenir ... Vous ne semblerez guère vous souvenir de moi ... Nous nous serons pourtant déjà rencontrés autrefois ... Ça devait être en $1891 \ldots$ en 92 ou en $93 . .$. dans une pièce de Maeterlinck ...

Voix de A. - Je ne crois pas que c'était moi. Vous vous trompez.

(Michel Perrin, L'Année prochaine à Wiesbaden, 285-84.)

36 Je fais entrechoquer, trembler des citations, sous forme de rappels. On aura remarqué, en effet, que les textes ou passages rapportés ci-dessus, du simple fait de leur rapprochement, grincent. Ou détonnent. Comme intérieurement contaminés par leurs voisins. Altérés et corrompus par la charge mutuelle qu'ils constituent réciproquement. 
Mettez le parodié près de son parodiant, il paraitra faux, révélant, bien sûr, une fausseté - peut-on le dire ? - qu'il contenait en germe. Mettez le texte de type 2 près de son analogue de type 1, il en ressortira une double lumière fort crue : ce qu'il n'avouait pas - le travail parodique - devient flagrant. J'insiste : même les premiers de série ne ressortent pas indemnes de la comparaison, Alain Robbe-Grillet ne tient pas devant Perrin, et l'originalité dont il paraissait à l'origine investi cède devant la poussée imitative.

Or, de tels rapprochements ne sont rien moins que factices: la comparaison constitue le paysage mémoriel du lecteur. Je lis dans les connexions, je vis dans les concomitances. Texte à texte, détectant des sources et des emprises. Il me faut le miroir, détectant par-dessus l'épaule ce qui vient se jeter, de connu, dans mon visage ... Il me faut le report et le rapport, défaillances et contiguités : je ne puis croire à ce que je lis, sauf à le dénaturer du mieux que je peux ... Ainsi, par exemple, l'application du texte de type 2 à celui de type 1 se fait-elle de la même manière que le pastiche (avéré) de celui-ci : nécessairement et automatiquement pour la mémoire active où ils entrent. Je ne puis me défaire de confronter. La mise en parallèle des lectures que je fais jette au tas ce dont je me saisis : tout texte paraît déduit du précédent, dans la chronologie réelle de la production - un peu comme dans une Histoire de la littérature bien faite - ou dans la chronologie factuelle et aléatoire de la lecture. Le rapprochement discontinu infini des produits dans lire falsifie toute écriture. Et je ne méjuge, en ceci, ni de l'un, ni de l'autre.

Cette assimilation constante des textes par leurs usagers mêmes à d'autres dont ils ne se déduisent pas nécessairement " à l'origine, " cette parodisation accélérée de la littérature dans l'acte de lecture, s'appuie, pour le cas qui nous occupe - les parodies du degré second - sur un phénomène remarquable : la généralisation effrénée des procédés. Tout se passe comme si je ne pouvais bien saisir, bien comprendre, bien maîtriser et faire mien que par universalisation radicale des processus - ou bien, lâchons le mot, de l'objet de désir. Par là, je ne fais rien d'autre que de (me) profiler sa perte: le généralisateur, comme l'imitateur, est un destructeur; la mémoire aussi décompose tout ce qu'elle touche. La fatalité (?), en effet, veut que l'efficacité d'un procédé dépende de son "lancement» culturel: le tour ou la façon doit émerger de son contexte concurrentiel, il doit faire tache, attirer sur lui l'intérêt, susciter le plaire et l'envie. Il vaut, dès lors, d'être compris comme objet d'imitation. Or, la parodie est une imitation non concédée. C'est dans la mesure ou l'objet en question est proposé à l'imitation et où celle-ci est tentée de s'en saisir qu'il intéresse. Cependant, cette «imitabilité » des textes ne leur advient pas, loin de là, que de l'extérieur; leur " généralité, » leur schématisme, leur systématisme, leur radicalité, leur typicité, leur rationalisation sont, à cet égard, de puissants facteurs. En un mot, le taux de cohérence apparente d'un texte, le caractère ostentatoire de son écriture, définit aussi son taux d'imitabilité (comment bien refaire ce qui se dissimine et se relance sans fin, y compris dans les sens contradictoires qu'il ne paraît pas comprendre ?). Or, conclusion facile, cette prestation - cette simplification, cette clarification - signifie aussi la dévalorisation de l'objet de désir : eu ou rendu, le voici perdu ... La dépréciation guette tout ce qu'on imite pour l'avoir convoité. Dilemme : laisserai-je intacte - intouchable inconnue - cette parole première qui me convient et qui me fait défaut ou la prononcerai-je à mon tour? Ne vois-je pas qu'elle m'échappe dès qu'elle obéit aux nécessités (primitives) de ma bouche, dès que je l'ai sur la langue? Non Chrysostome, je ne puis bien parler en Dieu... 


\section{La relance parodique indéfinie} appréciable, afin de se faire lire, ceux ou celui qui l'avaient précédé dans la série. Il ravive et renvoie, détourne et rejoue, ajourne aussi, chacun des termes, sémantiques ou stylistiques, de la perception.

Nous nous tenons, ici, proprement dans la modernité. Post - ou pas, disons que celle-ci commence au double, au dédoublement, à la doublure, à la duplicité, «quand la croyance à l'Un a fait long feu. $»^{4} \mathrm{Je}$ constate que nous voici à l'ère de la pléthore: trop de mots, trop de sens, encombrent nos circuits; nous sommes des engorgés. Nous écrivons, par suite, comme par expectoration. ${ }^{5}$. Ou encore: "La dérive [qui est le mouvement propre des êtres] ne se révèle-t-elle pas alors, a posteriori, comme une vaste conduite d'échec où la conquête d'une forme fixe et définitive est à chaque fois remise à plus tard et s'autodétruit lors de son inscription même dans le 'support' en dérive? Echouage, chute. Nous sommes tous en dérive, et plus précisément en son étape finale, car nous avons péché et sommes déchus » (Klébaner, 165). Dans l'univers de la pléthore, donc dans celui du manque. Manque de moi, trop de tous, trop de miroirs, trop de désirs, trop de personnes.

\section{Thèses}

41 Ecrire en arrive à un point de saturation telle gu'il ne peut être question que de récrire pour ce faire. Imitation généralisée obligatoire dans l'océan des cultures. Inflation des inflations. Emballement irrésistible des processus. Ecrire en arrive à ce point d'être nécessairement la parodie - la reprise en humour - sue ou pas, inquiète ou pas, d'autres discours présents, par masses ou par grands pans. J'écris dans la bibliothèque : je suis son rat songeur! Du même coup, écrire est nécessairement autoparodique de luimême : je ne saurais pas ne pas immanquablement, pour le dire, m'imiter.

\section{Lectures}

Et de nouveau :

Une table, une fenêtre, une table près d'une fenêtre, et la vue, les vues.

Le conservateur l'a dit, ne pourrais-je garder qu'un seul tableau, celui-ci: le montant (frame), les battants (leaves), les traverses (crossbars), l'accoudoir (sill), les poissons rouges (goldfish). Ouverte sur combien de paysages, de baies, de cours, de rues? A des années d'écart, les mêmes motifs, les mêmes entrelacs de l'appui imposent leurs lys renversés au carrefour bondé et au jardin avec son jet d'eau. Les voitures s'alignent en files régulières, dans l'attente du feu vert. Les allées de sable blanc se croisent au bassin. Et combien de feuilles blanches, quadrillées, étalées alors, vainement ? Puis, entre la table et la fenêtre (but that's what Virginia Woolf is all about, (my dear)).

- Jacob! Jacob!!

A New York, par exemple, tel matin d'Indépendance Day, sur la terrasse de l'ambassadeur, que tu aies lu, parcouru, trente pages du Portrait of a Lady, peut-être, aux yeux ces lunettes rondes, brunes, du boulevard Saint-Michel avec Bill, quinze jours auparavant (ou bien si plus tard, si une autre année ?). Et l'intérieur, soudain, la chambre fraîche, le brait du ventilateur, ses hélices, l'ombre, courant d'air, et la tristesse des parades invisibles, au loin, mais on les entend encore, la musique, les cris, par intermittence, un peu étouffés maintenant, au gré d'un vent infime, ou du trafic. Les rideaux sont poussés en avant, à travers la baie entrebâillée. Ils refluent 
d'un seul coup et flottent vaguement, assez haut, loin du sol. L'intérieur, le lit défait, les valises béantes, le bureau plat avec à droite, accumulé, le courrier de l'absent. Une carte postale, au sommet de la pile, représente la gare routière de Boise. La moitié gauche de son verso ne porte que deux mots, LOVE, de biais, en lettres majuscules, et la signature, Michaël. Imaginant, imaginant. Le Sud, l'avenir, la dernière ligne, l'orage en février. Le soleil apparaît dans une éclaircie de quelques minutes. Ou bien à Calcutta, mais alors si jeune vraiment, sur le balcon du consulat, fauteuil d'osier qui va s'évasant, beige et vert, aux ornements compliqués, balustrade de pierre trop blanche. Il le voit quitter l'allée de lauriers roses, faire quelques pas vers les tennis déserts à cette heure, en cette saison. La bicyclette d'Anne-Marie Stretter est appuyée au grillage qui les entoure et qui confère aux joueurs, s'il y en a, une réalité tremblotante. Ou bien elle est tombée, sans doute elle est tombée. «Ainsi, chez cet enfant, des choses couvaient, dit-elle, des choses qui ne ressemblaient pas à celles que nous attendions de lui, nous qui croyions le connaître. Qui aurait cru? » Les voix, à cette distance, ne se laissent pas identifier.

(Renaud Camus, Passage, 9-11.6)

La cour, en forme de trapèze, est entourée de hauts murs, sauf sur un de ses côtés étroits qu'occupe une double grille de fer peinte en blanc mais dont les charnières sont rouillées. Les murs sont de taille et de matériaux disparates. La cour est semée de gravillons qui crissent sous les pas de l'enfant. Au-dela de la grille, c'est une allée de tarmac que borde à droite le long pignon aveugle, dalles coulées d'un bloc, d'un hangar gris; à gauche, se trouve une suite de niches obliques fermées par des portes coulissantes en tôle, garages pour automobiles de pauvres. Au bout de l'allée, lui étant perpendiculaire, une rue étranglée à l'ombre des bâtiments fades de la cartonnerie.

De la table où l'homme écrit, on ne voit pas la rue.

Parfois, très rarement, une petite fille s'avance dans la cour et danse avec lenteur sur une musique connue d'elle seule. Elle est gracieuse, et tourne sur elle-même portée par ses jambes minces, entraînée par une douce rondeur de gestes. L'homme a moins froid, lui semble-t-il, mais l'enfant disparaît trop vite, avant qu'il ait eu le temps de retenir en sa mémoire brumeuse les figures simples et étranges de la danse. L'homme a rêvé, peut-être.

(Jean-Claude Pirotte, La Pluie à Rethel, 17.7)

Rome, en ce point de la légende, place, en dyptique, deux hé race, ou la carrière de Servius Tullius et l'institution $d$ 3 ensembles épiques laissant de côté d'autres mythes histori doué, d'autre part la guerre terminée, non pas de Cocles sép arce qu'il est le seul à appartenir complètement l'autre jur manique et en Baltique et ce correspondant figure justement. $\mathrm{n}$ aitios logos en liaison avec le temple de Jupiter Stater $\mathrm{d}$ $t$ l'histoire romaine commence. On voit aussi ce que cette st pays européens l'Arabe, Le Housar, le Juif et que notamment oulait pas de ces gendres sans opes et n'attachait les Sabin maniéré de dot, comment il inspira a Romulus dans ton coeur, Rome et cum generis suis auitas opes pro dote sociant, parta membre de la même race humaine sang et leur lignée villes d (ibid., XVI, 1933, co. 417, s v. Mucius Scæuola) a fait quivalence « Viçve Devâh = les 33 dieux » Rig-Veda, IX, 92 Oroastre pour l'humanité, Tishtriya pour les étoiles, etc), un ormeau pour cible, il propose des joutes de javelots à nu corrélation avec un tableau économique, la fortis Etruria ( ...) se prêter à tous les tête-à queue même, jugés opportuns Rome et la geste des 4 rois préétrusques, on reconnaît le mê veraineté, ceux que l'Inde, et déjà les Indo-Iraniens, comme rencontre (cette) onomastique n'enseigne pas grand-chose... texte,- mais dont on ne tient pas compte comme si Louve et 
genre social double romain « rex-Flamen » : et je viens de sou

lieu à de longues discussions sur lesquelles je reviendrai $t$

que, par conséquent, cette rencontre onomas - dénie-t-on aux

ls le sont ici dans l'ordre canonique ascendant des fonction

suiv., et l'article de St. Weinstock, s. v, Titii Sodales, da

17. Cette symétrie a été ensuite perdue de vue :J M.Q.II, pp

cussion de J M Q II, pp. 118 et suiv : améliorée, grâce à $M$.

(Denis Roche, Dépôts de savoir et de technique, 32. ${ }^{8}$ )

soi-même. Comme en un jeu: le discours ne provient pas d'une source authentique avérée - d'un moi autoritaire - mais de l'ensemble flou qui lui est réellement propre : Phrases, autant de flèches dans sa tête. Occupé d'écrire, le voici qui repasse des mots et ne peut que se voir à sa table précisément en train de le faire : j'écris que j'écris. « Mais lui a poussé sa table loin de la fenêtre et n'a plus pour vue que les feuilles blanches, quadrillées, et celle où s'interrompt, en son milieu, une ligne de caractères serrés, égaux, étonnamment réguliers. De même, mais dans l'autre sens. A la fin du premier jeu, dans un simple, le relanceur devient le servant, et le servant relanceur. A partir de là, les versions divergent» (Camus, 205), la scène reproduit le mouvement de reproduction qui l'anime. «On n'arrête pas de ressasser les mêmes vocables, » dit l'un (Pirotte, 93) ; «Je suis pris dans ce vaste ensemble d'ourlets successifs de pensée (...) le forcé-machine mais dans un tout autre ordre, autres dispositions, charné à saturer (...) Tu écris, donc tu penses; tu penses, donc tu techniques, » dit l'autre (Roche, 59, 63). Or, la réfection évide celui qui l'accomplit. Son rabot à la main, le voici posant comme un artiste, au lieu des auteurs qu'il détrousse, mais dénué d'une auréole : le ressasseur est le négateur de lui-même. Essence autoparodique de tous les Bouvard auteurs. Celui qui parle par discours interposés dissipe inévitablement l'esprit de sérieux dont pourtant il s'investit pour écrire. A répéter, me voici fondu dans les mots que je prends, parodiant, mais figé, par choc en retour, dans la posture timide du graveur ou du postier, adressant et grattant les mêmes inutiles messages que ceux-là qui me sont envoyés, je vois. Il est deux: il n'est pas sérieusement deux: c'est un rôle, il le dénature dans la mesure même où il s'en investit.

Supplément de parodie. La répétition parodique en arrive, immanquablement aussi, à ce point de rupture où parodié et parodiant se résorbent et font jaillir - lâchons le mot! - du sens. Comme un (obscène) inédit de signification. Car on ne saurait, apparemment, parodier ni sans fin, ni sans reste. Déception - ou soulagement ! - de ce qui paraît là au fond des corbeilles de réécriture! La redite assidue rate ou bute et aboutit à faire (heureusement?) ressortir le geste qu'on n'attendait pas, la trace, ou le motif, ou la vue, énormément caché, du sujet écrivant. Là où il est ressort des mots qu'il retire de la bouche des autres. Du fond obscur des répétitions une voix soudain se fait entendre. En somme, le moi transpire, mais c'est, par délégation, dans le délai, increvable et navrant.

Renaud Camus ? Le texte sidère, à travers le filtre citationnel, une action homosexuelle. Du récit surgit du fond des reprises pour faire saillir, substitutivement, ce qu'il ne paraissait pas ou n'osait pas dire : «Deux garçons le suivent, le dépassent, inventent autour de lui, d'un passage l'autre, les entrechats d'un ballet de plus en plus précis et se rapprochent, le touchent, le caressent (...) $(22,24)$.»

Jean-Claude Pirotte? Un moi dissous et dilué, ineffaçable pourtant - il navre - se dit sous métaphore. Même s'il s'ignore et se tait via redites, la fameuse pluie du Nord le 
pleut bien, elle: «Je voudrais ne produire qu'une averse de mots (...). Sur Rethel la pluie ne cessera de tomber pendant des millénaires. Tu tiendras ton amour ton entier lové sur la poitrine, et l'on n'entendra que le bruissement des gouttes sur les ardoises du toit $(109,136)$.»

Denis Roche ? Un référent recule et ce qui reste d'écrit est le plaisir de son imaginaire disparition. Cependant, rien ne se gomme. Cependant, rien ne résiste à l'écriture. La femme-objet du texte revient du fond du texte, au fur et à mesure que je l'y fais tomber. J'entasse ses vues. Je suis ce moi pyramidal - ce menhir érigé - ce gigantesque dépôt de désir que la langue produit : «Ce peintre peint un tableau (...). Puis, quand il a fini, il fait venir une femme chez lui, rencontrée dans la rue, la fait se déshabiller et poser devant ledit tableau dressé contre le mur (...). Cela fait, il recule de quelques mètres et prend une photo de l'ensemble. Ensuite, d'après cette photo, il peint un nouveau tableau devant lequel il fera poser une deuxième fois la femme nue pour en tirer encore une autre photo, et ainsi de suite. L'opération consiste ainsi à peindre un tableau d'après une photographie représentant une femme nue devant un tableau qui, luimême, représentait une photographie d'une femme nue devant un tableau: mise en abîme, en force, de la photographie dans la peinture cette surface n'en finit pas de se dire telle. (Ceci avait lieu chez un ami, et je me souviens que j'étais très excité par les photos de la femme (...))»(102-103).

Tableau sombre, en fin de compte donc, puisque ce qu'un texte désirait chasser fait retour à même la représentation, parodique ou pas; du sujet fuse là où on ne l'attendait pas, émerge de tout discours, même aliéné; l'étrange étrangeté qu'il vise ne se maintient jamais bien longtemps dans sa bouche. Comme ces arbres que le jardinier émonde jusqu'au col sur les promenades : insatiables pylônes, contre toute attente, les voici reverdir ...

\section{Conclusif}

J'insiste sur quelques résultats divers, ou leçons, que je crois pouvoir tirer de ce qui précède :

1. Le parodieur est l'objet propre, quoique détourné, de sa parodie. On appellera parodie une opération de lecture (d'interprétation) menée par un sujet sur lui-même à propos d'un objet qu'il se donne à cette fin, les conditions de superlativation et de généralisation étant par ailleurs remplies.

«Le pasticheur n'est donc pas seul: pour être cru, il lui faut la complicité de ses lecteurs, » disait Caradec (11). C'est pourtant, en dernière instance, à lui-même qu'il s'adresse, les lecteurs ne figurant pour lui-même que le relais nécessaire à son opération. L'autre lui tend son miroir. Catharsis des parodies: le bon parodieur est celui qui se débarrasse bien de cet Autre qui l'assiège. Il s'épure par l'imitatif discours. Croit-il. Le voici « propre » d'avoir congédié " dans le miroir parodique, " "en autrui, » par le biais d'un lecteur qui confirme, en riant, la justesse de son acte, toute l'impureté requise du parodié. Du bon petit rejet parodieur et de sa victime : à l'index figure nécessairement, par chance, cela qu'il me faudrait être si cela n'était pas déjà occupé.

2. Instabilité des opérations parodiques. Ni le sujet, ni l'objet, ni les moyens, critères ou marqueurs de la parodie ne sont stables ou certains. Pas plus au cours de l'Histoire des cultures que pour un temps donné : l'unité de savoir, d'écriture ou de lecture ne se rencontre pas. Par conséquent, tout est possible, parodiquement parlant, la diversité 
règne et l'indétermination des processus aussi : le parodié engendré par son parodiant (Vernet, 51), le parodiant à parodié inconnu, le parodié sans parodie, etc. Notre mémoire fonctionne apparemment comme une grande patine: tout y repasse, s'y reverse et revient. Sous bien des formes. Sous des formes extrêmes, offusquantes, repoussantes ou gaies. Onirisme parodique: ce que je me suggère en dormant (en écrivant), je me le repasse afin de m'en passer l'envie. Mais, ce faisant, le voici qui s'incruste : le parodieur est en perte d'être, de plus en plus évidé par ce qu'il dit revenir, avec horreur, à l'Autre. Désespoir du parodieur (de l'écrivain à bout d'écriture) : ayant tout éliminé de ce qu'il jugeait ne point bien convenir à sa nature, le voici épuisé, évacué, excisé de lui-même : tel aussi bien mort que triomphant.

3. Soyons divers; soyons tout ce que nous sommes. La réflexion sur la parodie, un peu plus, nous ferait croire qu'il existe deux sortes de discours, les bons et les pas bons, ceux qui conviennent et ceux qui mentent, les vérités, d'un côté, et les faussetés, de l'autre. Comme si le sujet avait (positivement) le choix entre un mal-dire et un bien-dire et comme s'il ne se tenait véritablement et authentiquement que là - du côté de l'assertion franche, droite et propre - du côté de la singularité - en un centre infrangible et pur. Même un homme comme Godard, il est vrai que c'est à propos de Je vous salue Marie, convient de ceci : "J'ai toujours un double discours ». Evidence : un sujet n'est pas commutable en un discours - en l'un quelconque de ses discours. Disparate, il l'est. Divers, il l'est. «Nous sommes ainsi ni d'où ni de vers quoi. Nous marchons tous dans la nuit un fanal à la main. Mais l'absence de point fixe nous est une grande ouverture. Et que le centre soit avec nous, et qu'il soit lumineux, notre prodigieux foyer » (Klébaner, 37). Emprunter plusieurs fréquences. « Tout a été dit, tout pourrait être dit encore. Un homme arrive et découvre l'objet échoué. Il se baisse, le ramasse, le considère. Son regard s'efforce de lui donner une totalité intelligible tandis que ses mains le parcourent. Mais ni envers ni endroit, ni haut ni bas, ne donnent à la conscience l'occasion d'exercer son pouvoir d'ordonnancement » (166).

Je revendique non seulement le droit de me contredire - facile ! - mais encore celui de maintenir ensemble le propre et 1'impropre, en un, de ce que je dis. La parodie peut servir à cela : affirmer tout en paraissant nier, nier tout en paraissant affirmer, affirmer d'une affirmation inégale, poursuivre sans fin dans la méprise, dans l'insu, aussi bien que dans le connu par dédoublement des voix nombreuses de tout le spectre des langues. Janus, guerre et paix, pile et face, je me trouve être ainsi toujours le gagnant sûr de mon discours.

4. Je parlerai de faux caméléon. Le faux caméléon est une bête étrange : il mime sa proie (sa mouche), d'une part, de façon à pouvoir s'approcher d'elle sans qu'elle y prenne garde, d'autre part, de façon à pouvoir échapper à d'autres prédateurs de plus grand format, ses semblables, ses frères. Je dis «faux caméléon, » car un caméléon ne possède, en vérité, aucune identité : toujours projeté vers un autre à saisir dont il diffère, il ne se l'adjuge et ne lui échappe qu'en parvenant à lui ressembler.

C'est au nom du propre qu'il guette ses mouches; c'est au nom du propre que, pour capter l'autre, il mue. Nous le voyons céder sa nature eu égard à sa nature. Il s'oblitère, c'est qu'il devient lui-même. Ainsi du parodieur, je crois, à la langue longue, mobile, rapide et courbe. 


\section{BIBLIOGRAPHY}

Bataille, Georges (1970), Cuvres complètes, I, Paris, Gallimard.

Camus, Renaud (1975), Passage, Paris

Caradec, François (1971), Trésors du pastiche, Paris, P- Horay.

Deguy, Michel (1984), « Limitation ou illimitation de l'imitation. Remarques sur la parodie. » In GROUPAR, éd., 1934, Le Singe à la porte. Vers une théorie de la parodie, New York, Peter Lang, 1-11.

Derrida, Jacques (1978), Eperons, Paris.

GROUPAR, éd., (1984) Le Singe a la porte. Vers une théorie de la parodie. New York, Peter Lang.

Houdebine, Anne Marie (1984), « Parodie et identité », in GROUPAR, éd., Le Singe a la porte. Vers une théorie de la parodie. New York, Peter Lang 57-65.

Klebaner, D. (1978), Poétique de la dérive. Paris, Gallimard.

Leroux, Gaston (1961), La Double vie de Théophraste Longuet. Paris, Minuit.

Lista, G. (1984), Le Livre futuriste. Paris.

Montaut Amie (1984), «L'Excès et le pas-tout. Pas l'origine : la vérité, » in GROUPAR, éd., Le Singe à la porte. Vers une théorie de la parodie. New York, Peter Lang, 143-50.

Nodier, Charles (1977), Histoire du Roi de Bohème et de ses sept châteaux (Première édition, 1830), Paris, Editions d'Aujourd'hui, Collection « Les Introuvables. »

Olbrecht-Tyteca (1974), Le Comique du discours. Paris.

Perrin, Michel (1971), L’Année prochaine à Wiesbaden. in François Caradec, éd., Trésors du pastiche.

Paris, P. Horay.

Pirotte, Jean-Claude (1982), La Pluie à Rethel. Paris.

Ray, Jean (1971), Le Mystère de la forêt, in François Caradec, éd., Trésors du pastiche, Paris, P. Horay.

Robbe-Grillet, Alain (1961), L’Année dernière à Marienbad. Paris, Minuit.

Roche, Denis (1980), Dépôts de savoir et de technique. Paris, Seuil.

Terrail Ponson du (1985), Les Millions de la Bohémienne, in Nathan, Michel, éd., Anthologie du roman populaire, 1836-1918. Paris. Maison Générale d'Editions.

Vernet, Max (1984), « Situation de la parodie, » in GROUPAR, éd., Le Singe a la porte. Vers une théorie de la parodie. New York, Peter Lang

\section{NOTES}

1. Voir GROUPAR, éd., Le Singe à la porte.

2. "La terreur ainsi faite épuise promptement la terreur": citation de Claude Lefort, in GROUPAR, éd., Le Singe à la porte, 84

3. G. Raillard, à propos de Max Ernst et de Raymond Roussel, in La Quinzaine Littéraire 437, 19.

4. M. Vernet : "Tout ce qui est second répète et dégrade, l'entropie est irréversible"(46).

5. L'auteur donne la liste, fort longue, de ses inspirateurs et note encore que:"Le reste est constitué, pour la moitié au moins, de citations tirées d'écrits antérieurs de 1'auteur"(207). 
6. Commentaire préliminaire de l'auteur: "Il me reste cette chose à accomplir: user la parole. Me confondre avec elle dans l'épuisement des journées" (11).

7. On peut lire ceci sur la technique employée: "Répéter à l'infini, en étant libre de m'arrêter à n'importe quel moment, une même longueur de texte (...) une même longueur d'écriture déjà faite" (107). Je renvoie p. 31 pour les livres - souches de passage qu'on vient de lire.

8. Déclaration reproduite dans L'Autre journal 2 (janvier, 1985), 15.

\section{AUTHOR}

\section{CHARLES GRIVEL}

Université de Heidelberg 